Supporting Information for:

\title{
Probing the Dissolved Gas Concentration on the Electrode through Laser-Assisted Bubbles
}

\author{
Kota Ando, ${ }^{\mathrm{a}}$ Yoshiharu Uchimoto, ${ }^{\mathrm{b}}$ and Takashi Nakajima ${ }^{\mathrm{a}}$ \\ anstitute of Advanced Energy, Kyoto University, Gokasho, Uji, Kyoto 611-0011, Japan \\ ${ }^{b}$ Graduate School of Human and Environmental Studies, Kyoto University, Sakyo-ku, Kyoto 606-8501, Japan
}

*Corresponding author: nakajima@iae.kyoto-u.ac.jp 
(a)

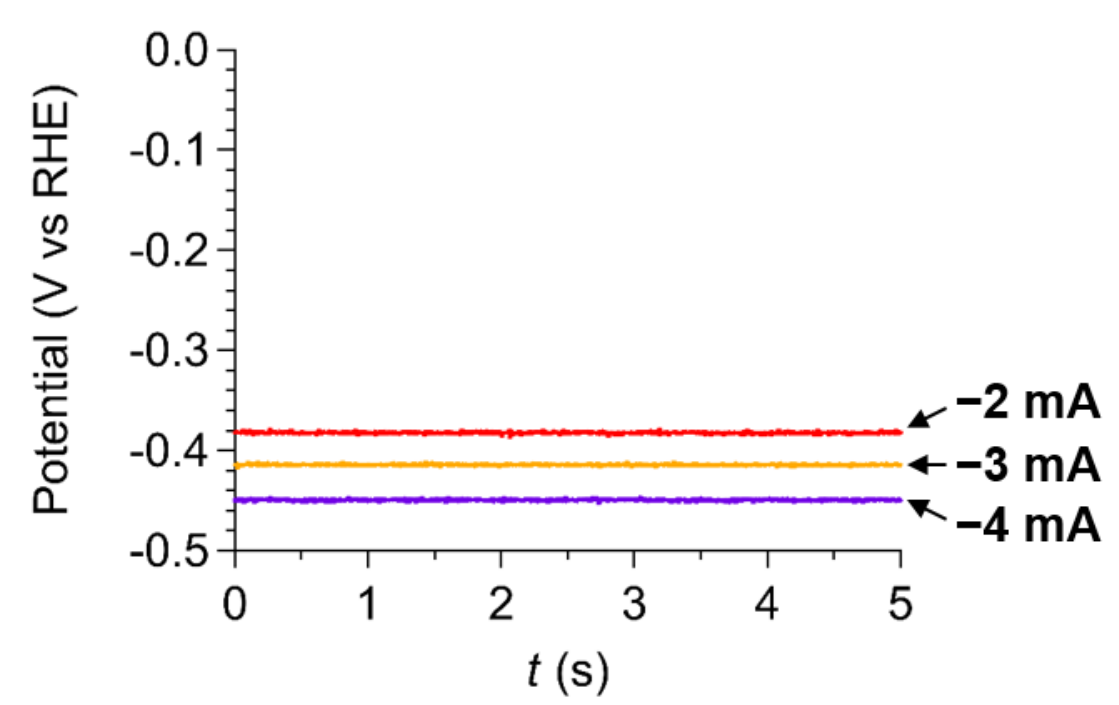

(b)

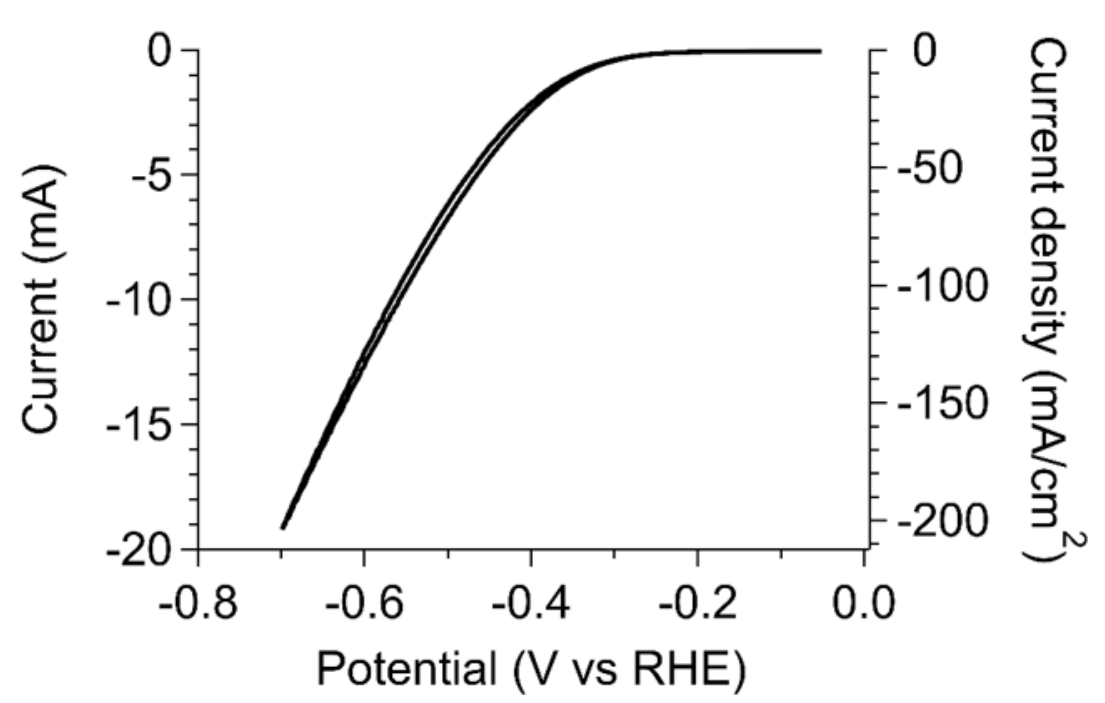

Figure S1. (a) Potential changes (without IR-correction) of the Ni wire electrode as a function of time during the measurements of laser-assisted bubbles. (b) A cyclic voltammetry curve (without IR-correction) of the $\mathrm{Ni}$ wire electrode at sweep rate of $50 \mathrm{mV} / \mathrm{s}$. 
(a)

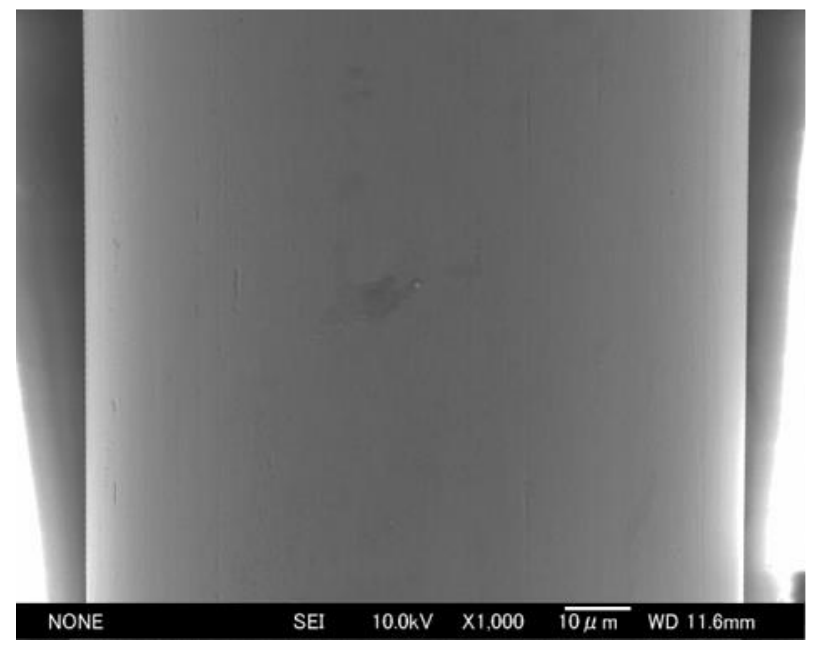

(b)

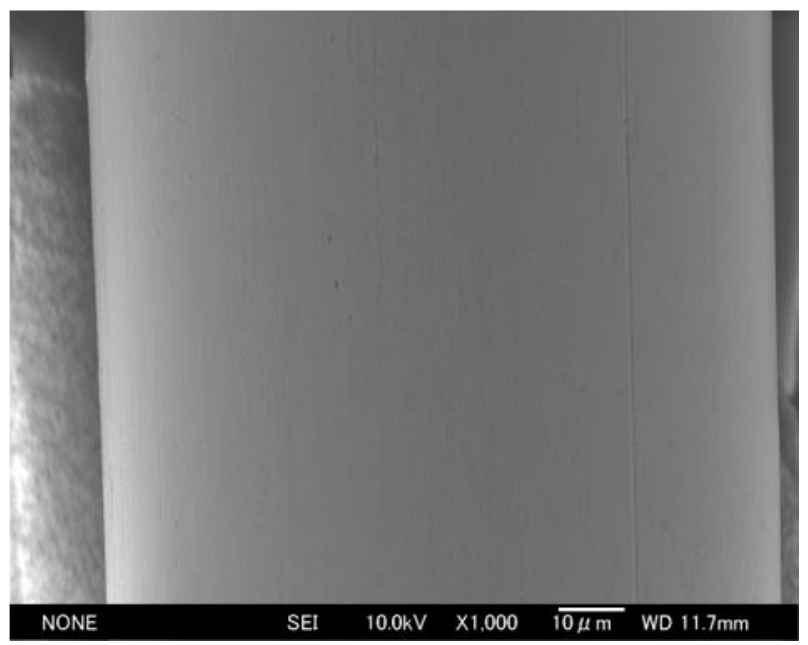

Figure S2. SEM images of the surface of a commercial Ni wire (a) before laser irradiation and (b) after laser irradiation of 100 shots at the laser fluence of $15 \mathrm{~mJ} / \mathrm{cm}^{2}$ in pure water. 


\section{Image analysis}

For the image analysis of laser-assisted bubbles on the electrode we develop a new program based on the image analysis VIs installed in NI LabVIEW (NI). Note that this program is different from the previous one we have developed for the bubbles in proximity to the electrode [1], because the latter does not work for the bubbles which are on or very close $(<15 \mu \mathrm{m})$ to the electrode. Within a very short time after the birth, initially flat laser-assisted bubbles grow and become almost spherical on the electrode (Movies S1-S3), and the newly developed program can analyze the one-piece image of "a bubble on the electrode", to obtain the bubble growth rates through the following three steps (Figure S3): (1) identify the lower-boundary of the electrode in the first frame (in which the laser pulse is turned on), and the bump's contour in the subsequent images, (2) evaluate the vertical distance of the respective bumps with respect to the lower-boundary of the electrode, and (3) determine the heights of the respective bumps through peak detection. By repeating steps (1)-(3) for all frames of the movies, we obtain the bubble growth rates. As we can see in Movies S1-S3, the shape of the laser-assisted bubble is hemispherical up to the height of $\sim 6 \mu \mathrm{m}$, but after that it becomes practically spherical with a very small contact area on the electrode surface. When the bubbles grow to the height of $\sim 15.6 \mu \mathrm{m}$ they start to detach from the electrode. Therefore, the most convenient size of the laser-assisted bubbles for the analysis of growth rate is $6-15 \mu \mathrm{m}$ in terms of the bubble diameter. 
Step 1: Contour detection
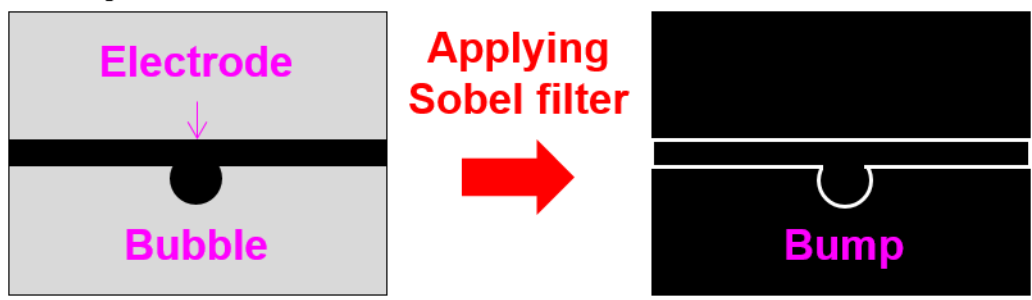

\section{Step 2: Evaluation of vertical distance}
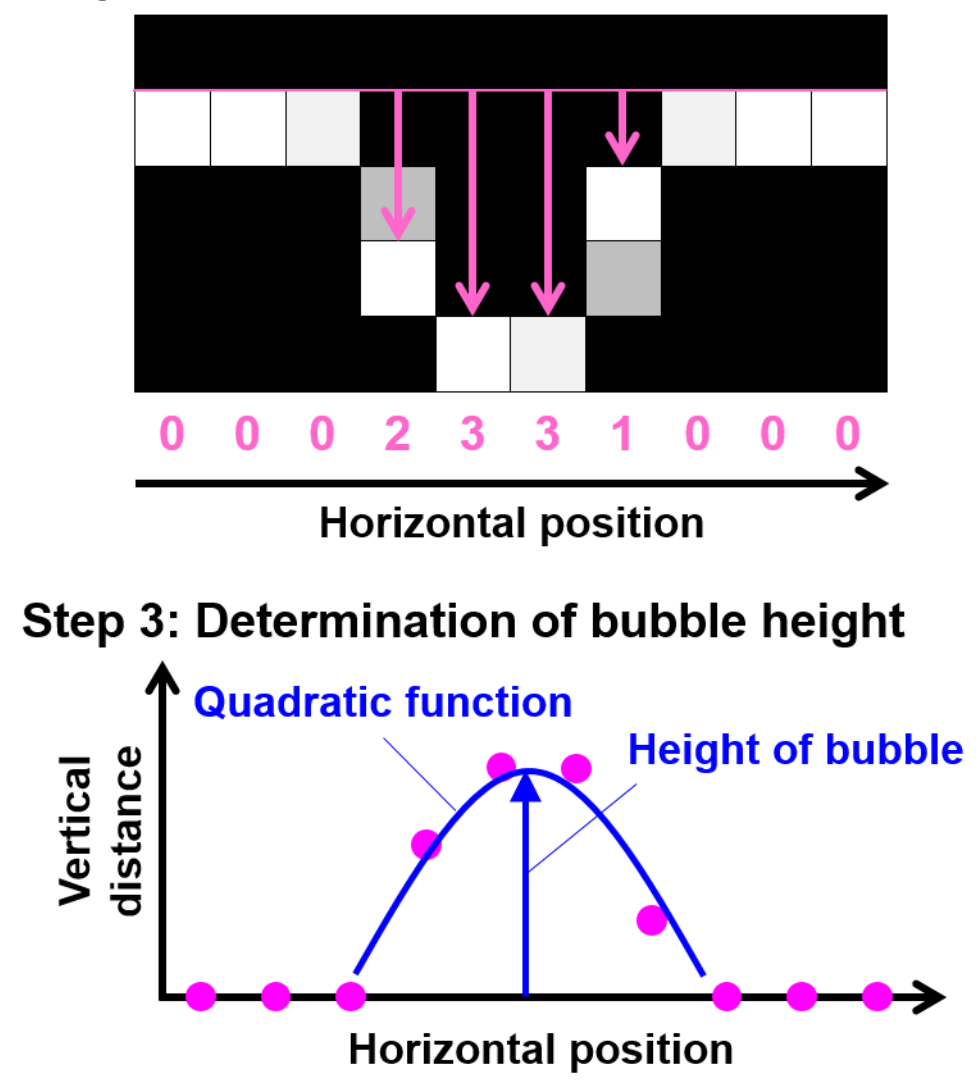

Figure S3. Illustration of the image analysis of laser-assisted bubbles on a wire electrode. [Step 1] Contour detection of the electrode and a laser-assisted bubble by applying the Sobel filter to the raw image. Since the electrode surface and the bubble appear as a one-piece image, the bubble looks like a bump of the electrode in the image. [Step 2] Evaluate the vertical distance of the respective bumps from the lower-boundary of the electrode. [Step 3] Determine the height of the respective bumps through peak detection. By repeating Step 1-3 for all frames, we obtain the bubble growth rates. 


\section{Evaluation of dissolved gas concentration from the growth rates of bubbles}

\section{Epstein-Plesset equation}

In this section we briefly describe a diffusion model [2] we have employed in this work. Usually a diffusion model is used to predict the bubble growth under a given concentration of the dissolved gas in a solution, but we use it the other way around: we use the model to obtain the concentration of the dissolved gas from the experimentally measured growth rate of a bubble on the electrode.

The time evolution of a stationary bubble with a radius, $R$, in a supersaturated solution with a uniform dissolved gas concentration can be described by the well-known Epstein-Plesset (EP) equation [2], which reads

$$
\frac{d R}{d t}=D \frac{C-k_{\mathrm{H}} P_{\mathrm{B}}}{n_{\mathrm{B}}}\left(\frac{1}{R}+\frac{1}{\sqrt{\pi D t}}\right)
$$

where $D$ and $C$ are the diffusion coefficient and dissolved gas concentration, respectively, with $k_{\mathrm{H}}$ being the Henry constant, and $P_{\mathrm{B}}$ and $n_{\mathrm{B}}$ the pressure of the gas and number density of the gas molecules inside the bubble. The time-dependent feeding term (the second term) on the right-hand side of the EP equation, eq $\mathrm{S} 1$, is negligible if $R^{2} \ll \pi D t$ or equivalently $\mathrm{d} R^{2} / \mathrm{d} t \ll \pi D$, where $\pi D \approx 12 \times$ $10^{-9} \mathrm{~m}^{2} / \mathrm{s}$ since $D=4.2 \times 10^{-9} \mathrm{~m}^{2} / \mathrm{s}$ for hydrogen molecules in water [3]. This is indeed our case. Under this condition, we may discard the second term on the right-hand side of eq S1 to obtain the simplified EP equation, which reads,

$$
\frac{d R^{2}}{d t}=2 D \frac{C-k_{\mathrm{H}} P_{\mathrm{B}}}{n_{\mathrm{B}}} .
$$

Note that $\mathrm{d} R^{2} / \mathrm{d} t$ instead of $\mathrm{d} R / \mathrm{d} t$ appears on the left-hand side now, and the bubble radius, $R$, does not appear on the right-hand side. Therefore, once we experimentally measure the growth rate of the 
squared value of the bubble radius, $\mathrm{d} R^{2} / \mathrm{d} t$, we are able to obtain the dissolved gas concentration, $C$, by referring to the simplified EP equation, eq S2, since the constants, $D$ and $k_{\mathrm{H}}$, are known [3, 4], and $P_{\mathrm{B}}$ and $n_{\mathrm{B}}$ can be easily estimated as follows. By taking into account the influence of the surface tension, $\gamma$, for the surface tension at the gas-liquid interface, $P_{\mathrm{B}}$ and $n_{\mathrm{B}}$ are given by

$$
P_{\mathrm{B}}=P_{0}+\frac{2 \gamma}{R}
$$

and

$$
n_{\mathrm{B}}=n_{0}\left(1+\frac{2}{3} \frac{2 \gamma}{R P_{0}}\right)
$$

respectively, where $P_{0}$ and $n_{0}$ are the pressure and number density of molecules inside the gas with a flat gas-liquid interface, i.e., those of the atmosphere. Using $R=8 \mu \mathrm{m}$ and $\gamma=72 \mathrm{mN} / \mathrm{m}$ for the largest bubble radius and surface tension at the gas-water interface, the pressure difference between the inside and outside of the bubble (so-called Laplace pressure), $2 \gamma / R$, is calculated to be about $1.8 \times 10^{4} \mathrm{~Pa}$, which is $\sim 18 \%$ of the atmospheric pressure. Thus, even for the largest bubble radius we measure in this work the Laplace pressure plays an important role. The analytical solution of the EP equation, eq $\mathrm{S} 1$, with the Laplace pressure had been given in their original paper [2]. Note that eq S2 is identical with Eq. (34) in their paper [2] if the time-dependent term on the right-hand side of Eq. (34) is discarded. After ensuring that eq S2 and Eq. (34) in Ref. [2] are essentially identical we employ the analytical solution of Eq. (34) to calculate the temporal variations of $R^{2}$ for the bubble in the solution with uniform dissolved gas concentrations of $7.8-31.2 \mathrm{mM}$ by recalling $D=4.2 \times 10^{-9} \mathrm{~m}^{2} / \mathrm{s}[3], k_{\mathrm{H}}=0.78$ $\mathrm{mM} / \mathrm{atm}$ [4], $P_{0}=1 \mathrm{~atm}, n_{0}=40.9 \mathrm{mM}$, and $\gamma=72 \mathrm{mN} / \mathrm{m}$. The results are shown in Figure $\mathrm{S} 4$. As we 


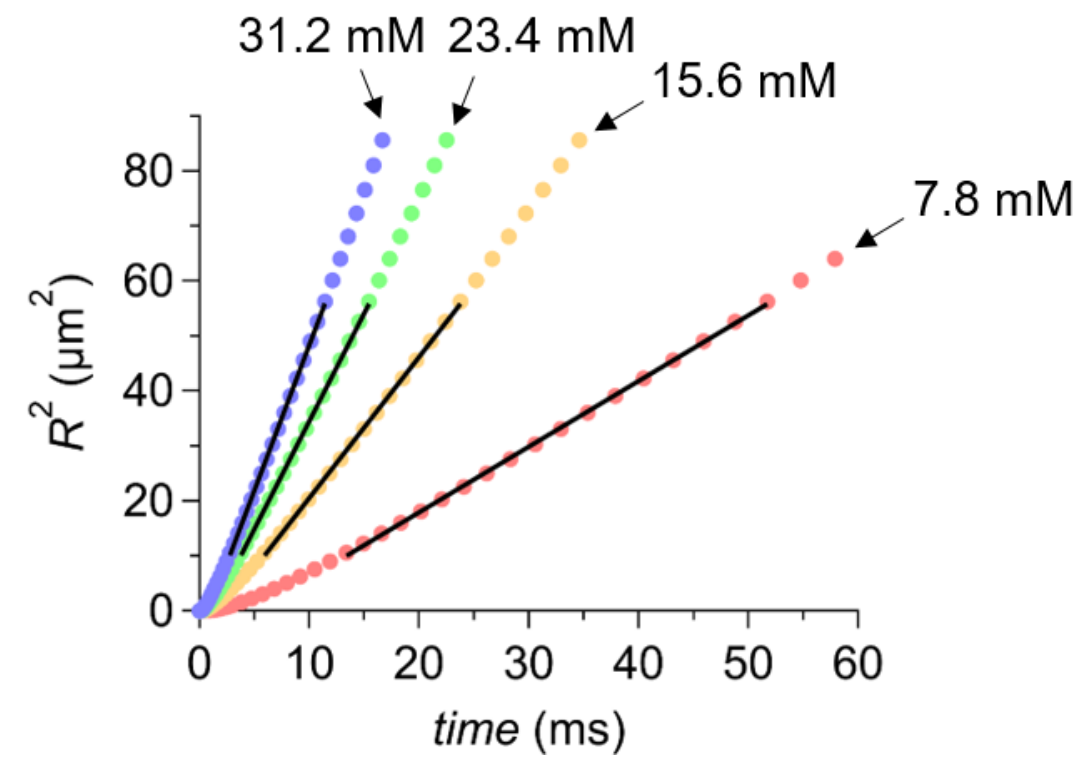

Figure S4. Temporal variation of the squared bubble radius in a supersaturated solution with a uniform dissolved gas concentration of 7.8, 15.6, 23.4, and 31.2 $\mathrm{mM}$, respectively, calculated by using the solution of eq S2 that takes into account the influence of the surface tension. The solid lines are the linear fittings in the size range of $10 \mu \mathrm{m}^{2}<R^{2}<60 \mu \mathrm{m}^{2}$, which can be approximated by the solution of eq S5 with $\alpha=0.85$.

see, $R^{2}$, for the range of $10 \mu \mathrm{m}^{2}<R^{2}<60 \mu \mathrm{m}^{2}$ or $3.2 \mu \mathrm{m}<R<7.8 \mu \mathrm{m}$, is linear with respect to time.

Known this linearity, we introduce a correction factor, $\alpha$, to approximately take into account for the

Laplace pressure. Thus, the simplified EP equation with the Laplace pressure correction read,

$$
\frac{d R^{2}}{d t}=2 D \frac{\alpha C-k_{\mathrm{H}} P_{0}}{n_{0}}
$$

For the range of $10 \mu \mathrm{m}^{2}<R^{2}<60 \mu \mathrm{m}^{2} \alpha=0.85$ is appropriate to include the influence of Laplace pressure, while we can set $\alpha=1$ to neglect it. Using eq S5, we can calculate the dissolved gas concentration, $C$, without/with the influence of Laplace pressure, and the results are shown in Table 1. 


\section{Reference}

[1] K. Ando, Y. Uchimoto, and T. Nakajima, Chem. Commun. 56, 14483 (2020).

[2] P. S. Epstein and M. S. Plesset, J. Chem. Phys. 18, 1505 (1950).

[3] H. Vogt, Electrochim. Acta 25, 527 (1980).

[4] R. Fernández-Prini, J. L. Alvarez, and A. H. Harvey, J. Phys. Chem. Ref. Data 32, 903 (2003). 